\title{
Latest developments on Streptococcus suis: an emerging zoonotic pathogen: part 1
}

\begin{abstract}
Mariela Segura* ${ }^{*, 1}$, , Han Zheng ${ }^{2, t}$, Astrid de Greeff $^{3}$, George F Gao ${ }^{4}$, Daniel Grenier ${ }^{5}$, Yongqiang Jiang ${ }^{6}$, Chengping Lu $^{7}$, Duncan Maskell ${ }^{8}$, Kazunori Oishi ${ }^{9}$, Masatoshi Okura $^{10}$, Ro Osawa ${ }^{11}$, Constance Schultsz ${ }^{12}$, Christian Schwerk ${ }^{13}$, Tsutomu Sekizaki ${ }^{14}$, Hilde Smith ${ }^{15}$, Potjanee Srimanote ${ }^{16}$, Daisuke Takamatsu ${ }^{10}$, Jiaqi Tang ${ }^{17}$, Tobias Tenenbaum ${ }^{13}$, Prasit Tharavichitkul ${ }^{18}$, Ngo Thi Hoa ${ }^{19}$, Peter Valentin-Weigand ${ }^{20}$, Jerry MWells ${ }^{21}$, Heiman Wertheim ${ }^{22}$, Baoli Zhu ${ }^{4}$, Marcelo Gottschalk ${ }^{1,+}$ \& Jianguo Xu ${ }^{2,1, \neq}$
\end{abstract}

First International Workshop on Streptococcus suis, Beijing, China, 12-13 August 2013

The first international workshop on Streptococcus suis, which is an important swine pathogen and emerging zoonotic agent, took place in Beijing, jointly organized by the Faculty of Veterinary Medicine, University of Montreal, Canada and the National Institute for Communicable Disease Control and Prevention, China CDC. The aim of the meeting was to gather together, for the first time, more than 80 researchers working on S. suis, from countries including China, Canada, Japan, The Netherlands, Germany, Thailand, the UK and Vietnam. This article, the first of a two-part report on this First International Workshop, reviews current aspects of the epidemiology and population genomics of S. suis, covers public health concerns and discusses questions about $S$. suis serotyping and molecular diagnostics.

\footnotetext{
'Faculty of Veterinary Medicine, University of Montreal, Canada

${ }^{2}$ State Key Laboratory for Infectious Disease Prevention \& Control, National Institute for Communicable Diseases Control \& Prevention, China CDC

${ }^{3}$ Central Veterinary Institute of Wageningen, The Netherlands

${ }^{4}$ CAS Key Laboratory of Pathogenic Microbiology \& Immunology, Chinese Academy of Sciences, China

${ }^{5}$ Laval University, Canada

${ }^{6}$ Academy of Military Medical Sciences, China

${ }^{7}$ Nanjing Agricultural University, China

${ }^{8}$ University of Cambridge, Cambridge, UK

${ }^{9}$ Osaka University, Japan

${ }^{10}$ National Institute of Animal Health, Japan

"Kobe University, Japan

${ }^{12}$ Academic Medical Center, University of Amsterdam, The Netherlands

${ }^{13}$ University Children's Hospital Mannheim, Heidelberg University, Germany

${ }^{14}$ University of Tokyo, Japan

${ }^{15}$ Central Veterinary Institute, The Netherlands

${ }^{16}$ Thammasat University, Thailand

${ }^{17}$ Nanjing General Hospital of Nanjing Military Command, China

${ }^{18} \mathrm{Chiang}$ Mai University, Thailand

${ }^{19}$ Oxford University Clinical Research Unit, Ho Chi Minh City, Vietnam

${ }^{20}$ University of Veterinary Medicine Hannover, Germany

${ }^{21} \mathrm{HMI}$, Wageningen University, The Netherlands

${ }^{22}$ Oxford University Clinical Research Unit, Hanoi, Vietnam

*Author for correspondence: mariela.segura@umontreal.ca

${ }^{\dagger}$ Chairs and organizers

${ }^{\ddagger}$ Contributing co-authors are in alphabetical order
}

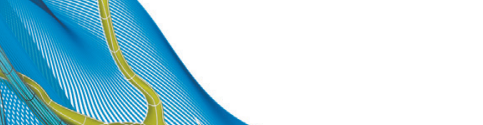

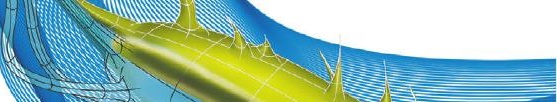

\section{(1)}

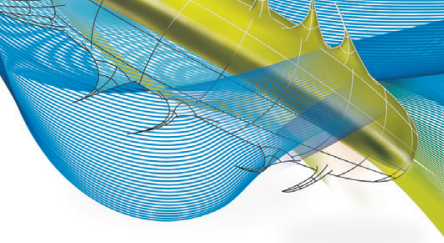

\section{KEYWORDS}

- emerging zoonosis

- epidemiology

- genomics • molecular diagnostics $\bullet$ public health

- Streptococcus suis 
Relevance of $S$. suis research

Streptococcus suis is a swine pathogen responsible for important economic losses in the swine industry worldwide. It causes a wide variety of diseases in pigs, including meningitis, septicemia and endocarditis. Among the 35 serotypes originally described based on capsular polysaccharide (CPS) antigens, serotype 2 is not only prevalent in swine diseases, but is also considered to be an emerging zoonotic agent causing meningitis and streptococcal toxic shock-like syndrome (STSLS) in humans [1]. S. suis has gained more attention since recent recognition of its high prevalence in human meningitis cases in southeast and east Asia, and reports of outbreaks that resulted in high mortality rates $[2,3]$. The emergence of $S$. suis as a human pathogen has led to an explosion of research. As of November 2013, 371 S. suis-related articles were published during the previous 5 years and more than 140 in the last 20 months (PubMed database). Here, we discuss current aspects emerging from the First International S. suis Workshop held in China in 2013.

\section{Advances in epidemiology \& population genomics}

The genome sequences of over 100 S. suis strains of different geographic origins have either been published or are available in public databases. Real-time genomic epidemiological evaluation of infectious agents, and in particular of those with potentially severe clinical outcomes, might limit detrimental effects of infections and prevent outbreaks. Dr Jianguo Xu (National Institute for Communicable Diseases Control and Prevention, China CDC) described a newly developed S. suis whole-genome sequencebased typing schema by which the minimum core genome of the species was defined. This method provides a clear division of groups containing human isolates and discriminates outbreak isolates, including those causing STSLS, from those causing sporadic, less severe disease [4]. This typing system will facilitate the application of genome data to S. suis epidemiology and surveillance. Using comparative genomics, Dr Baoli Zhu (Chinese Academy of Sciences, China) showed that $S$. suis type 2 has a polymorphic, highly mobile and mosaic genome. The $89 \mathrm{~K}$ pathogenic island, so far unique to Chinese STSLS-causing strains, was found to be partially included in other virulent and avirulent strains [5]. Dr Duncan Maskell (University of Cambridge, UK), again using wholegenome sequencing, described the population biology/structure of over 400 isolates from pigs in the UK and pigs and humans in Vietnam, which have detailed clinical histories or were isolated from tonsils of healthy pigs. Similar findings to those of $\mathrm{Dr} \mathrm{Xu}$ were reported in terms of there being extensive recombination. Data analysis using discriminant analysis of principle components identified particular single nucleotide polymorphisms that are associated with disease. Studies on S. suis infection in Thailand have mainly focused on human isolates. Dr Potjanee Srimanote (Thammasat University, Thailand) reported the genetic linkage between S. suis serotype 2 isolates from pigs and humans during the 2007 infection outbreak in Thailand. A strong correlation between virulence gene profiles, random amplified polymorphic DNA patterns and multilocus sequence types (STs) was observed, confirming $S$. suis transmission from pigs to humans. Multilocus STs distinguished four STs prevalent in this country: ST1, ST104, ST233, and a new ST336 [6].

\section{Advances in serotyping \& molecular diagnostics: the dilemma of $S$. suis serotypes}

S. suis serotyping is based on CPS composition and the use of antisera, which is expensive and time-consuming. There is also a need to complete knowledge on the diversity and evolution of S. suis CPS types. Dr Daisuke Takamatsu (National Institute of Animal Health, Japan) provided a comprehensive analysis of $S$. suis cps gene clusters. Albeit all CPS serotypes are synthesized by the Wzx/Wzy pathway, the number and combinations of $c p$ s genes differ among serotypes and, except for serotypes 1, 2, 1/2 and 14, all serotypes possessed serotype-specific gene(s). The evolution of the $S$. suis cps cluster diversity is the result of multiple gene transfer and recombination events, as well as small-scale mutations [7]. Interestingly, a large number of $S$. suis isolates, especially those from healthy pigs, remain untypable. Using whole-genome high-throughput sequencing, Dr Han Zheng (National Institute for Communicable Diseases Control and Prevention, China CDC) reported 12 new cps gene clusters among 'untypable' strains. The potential discovery of new serotypes remains to be validated.

Development of a practical and simple typing method for $S$. suis is desirable. Dr Chengping 
Lu (Nanjing Agricultural University, China) reported the development of serotype-specific PCR tests for nine different $S$. suis serotypes [8]. Dr Masatoshi Okura (National Institute of Animal Health) developed a multiplex PCRbased cps-typing method for the 35 described serotypes, composed of grouping and typing steps: a first PCR detects cps genes conserved in multiple serotypes (grouping PCR), followed by a second PCR that detects serotype-specific $c p s$ genes within each group. Since $c p s$ gene clusters of serotypes $1 / 2$ and 2 and those of serotypes 1 and 14 are almost identical to each other, the method does not discriminate between these serotypes [9].

Most PCR tests that are directly used with clinical specimens are directed against serotypes 2 (and 1/2) and 14 (and 1), responsible for the majority of human cases. Since PCR equipment is not always available in certain countries, Dr Prasit Tharavichitkul (Chiang Mai University, Thailand) described the rapid detection of these $S$. suis serotypes in streptococcal-positive hemocultures using the Quellung reaction with specific anti-CPS antiserum.

During the Workshop, a controversy arose regarding the taxonomy of $S$. suis strains. Serotypes 32 and 34 had previously been reported to belong to the species $S$. orisratti. PCR tests based on $g d h$ or $16 S$ rRNA primers are largely used to identify a field strain as $S$. suis. Dr Tharavichitkul reported that $g d h$ primers are less specific than those of $16 S$ rRNA [10]. Dr Ro Osawa (Kobe University, Japan) described a potentially more reliable PCR assay targeting the $\operatorname{rec} N$ gene, which encodes a DNA recombination/repair protein. He proposed that the reference strains of serotypes 20,22, 26 and 33, even if they are considered to be $S$. suis by the gdh or $16 S$ rRNA PCR tests, might not be $S$. suis based on DNA-DNA reassociation, as well as sodA- and recN-based phylogenies [11]. There is still a need for a consensus approach to define S. suis strains, especially for diagnostic laboratories. There is also a call for the complete genomic sequencing of the 35 serotypes to resolve this issue.

\section{Highlights on zoonotic \& public health concerns: the need of prevention campaigns}

The danger of S. suis for humans and the educational and preventive measures campaigns in Asian populations at risk were discussed during the workshop [12]. In Vietnam, consumption of raw or undercooked pork products has been related to large numbers of cases [2]. Dr Heiman Wertheim (Oxford University Clinical Research Unit, Hanoi, Vietnam) investigated the behavior of eating tiêt canh (raw pig blood) and found a lower rate of consumption in urban regions compared with rural areas. While some rural people start eating tiêt canh in their early childhood, most of them start to do so after getting married. Weddings are a common occasion where people are first exposed to this dish. In urban areas, people often eat tiêt canh mainly for breakfast in food shops. Interestingly, manual workers and sales persons were more likely to consume tiêt canh than office workers. To understand the potential risk of pig-derived products in the food chain, Dr Ngo Thi Hoa (Oxford University Clinical Research Unit, Ho Chi Minh City, Vietnam) investigated the presence of $S$. suis in pork byproducts collected from slaughterhouses and wet/retail markets in Vietnam, and found an $11 \%$ contamination rate with $S$. suis serotype 2 in internal organ samples. However, the potential of these isolates to cause disease remains to be evaluated [13]. Dr Kazunori Oishi (Osaka University, Japan) reported public health interventions in Thailand. Data suggest a high incidence rate $(6.2 / 100,000)$ of $S$. suis infection in the general population in 2010, mostly related to consumption of raw pork products. A risk assessment of human infection was disseminated to the local residents and the implementation of the Food Safety Campaign to 'stop consuming raw pork products' started in 2011 [14,15].

In contrast to Asian countries, human S. suis infection is considered a swine-related occupational disease in western countries. Infection rates in the general population and in-risk groups are poorly known because $S$. suis infection is not a notifiable disease. Dr Constance Schultsz (Academic Medical Center, University of Amsterdam, The Netherlands) reported that the annual incidence rate of 3-5 per 100,000 individuals at risk in The Netherlands is an underestimate. The National Institute of Public Health of The Netherlands recently placed S. suis among its top ten priority zoonotic pathogens for which increased awareness and disease surveillance is warranted. Serotype 2 was identified as the main cause of human infections, whereas serotype 9 is much more common in invasive porcine infections in that country [16]. 


\section{Future perspective: part 1}

The precise functions of each cps gene and CPS structures are still unknown for most serotypes. It is also unclear why specific serotypes or STs tend to cause diseases in swine and/or humans. For a comprehensive understanding of this important virulence factor, further studies with experts in different fields will be necessary. Interdisciplinary studies will also be required to uncover the complex serological interrelationships between serotypes $1 / 2$ and 2 and serotypes 1 and 14 . Finally, a major conclusion of the workshop is the need for an international consortium to form a consensus on $S$. suis identification at the species level.

In terms of public health, epidemiological surveillance should continue and communication campaigns through mass media, social and health workers and educational programs must be intensified in populations at risk.

\section{Acknowledgements}

The authors would like to thank the contribution of Huong $\mathrm{Vu}$ Thi Lan, Huynh Ngan Ha (Oxford University, Vietnam) and Laetitia Bonifait (Laval University, Canada).

\section{Financial \& competing interests disclosure}

Organization of the First International Workshop on Streptococcus suis was funded by the Canada-China collaboration program financed by the Canadian Institutes of Health Research and the National Natural Science Foundation of China (812111251). The authors have no other relevant affiliations or financial involvement with any organization or entity with a financial interest in or financial conflict with the subject matter or materials discussed in the manuscript apart from those disclosed.

No writing assistance was utilized in the production of this manuscript.

\section{References}

Papers of special note have been highlighted as: - of interest

1 Gottschalk M, Xu J, Calzas C, Segura M. Streptococcus suis: a new emerging or an old neglected zoonotic pathogen? Future Microbiol. 5(3), 371-391 (2010).

2 Wertheim HF, Nghia HD, Taylor W, Schultsz C. Streptococcus suis: an emerging human pathogen. Clin. Infect. Dis. 48(5), 617-625 (2009).

- Comprehensive review on the clinical and epidemiological features of the Streptococcus suis infection in humans in Asia.

3 Fulde M, Valentin-Weigand P. Epidemiology and pathogenicity of zoonotic streptococci. Curr. Top. Microbiol. Immunol. 368, 49-81 (2013).

4 Chen $\mathrm{C}$, Zhang W, Zheng $\mathrm{H}$ et al. Minimum core genome sequence typing of bacterial pathogens: a unified approach for clinical and public health microbiology. J. Clin. Microbiol. 51(8), 2582-2591 (2013).

- A typing system that facilitates the application of genome data to the fields of $S$ suis clinical medicine and epidemiology.

5 Wu Z, Li M, Wang C et al. Probing genomic diversity and evolution of Streptococcus suis serotype 2 by NimbleGen tiling arrays. BMC Genomics 12, 219 (2011).

- Deep comparative genomic analysis of the $89 \mathrm{~K}$ pathogenicity island.
6 Maneerat K, Yongkiettrakul S, Kramomtong I et al. Virulence genes and genetic diversity of Streptococcus suis serotype 2 isolates from Thailand. Transbound Emerg. Dis. 60 (Suppl. 2), 69-79, (2013).

7 Okura M, Takamatsu D, Maruyama F et al. Genetic analysis of capsular polysaccharide synthesis gene clusters from all serotypes of Streptococcus suis: potential mechanisms for generation of capsular variation. Appl. Environ. Microbiol. 79(8), 2796-2806 (2013).

- First complete description of $c p$ s loci of all serotypes of $S$. suis.

8 Wang K, Sun X, Lu C. Development of rapid serotype-specific PCR assays for eight serotypes of Streptococcus suis. J. Clin. Microbiol. 50(10), 3329-3334 (2012).

9 Okura M, Lachance C, Osaki M et al. Development of two-step multiplex PCR assays for typing of capsular polysaccharide synthesis gene clusters of Streptococcus suis. J. Clin. Microbiol. doi:10.1128/JCM.03411-13 (2014) (Epub ahead of print).

10 Le HTT, Sugiyama N, Duangsonk K, Tharavichitkul P, Osawa R. Phenotypic and PCR-based identification of bacterial strains isolated from patients with suspected Streptococcus suis infection in northern Thailand. Jpn J. Infect. Dis. 65(2), 171-174 (2012).

11 Le HTT, Nishibori T, Nishitani Y, Nomoto R, Osawa R. Reappraisal of the taxonomy of Streptococcus suis serotypes 20,22, 26, and 33 based on DNA-DNA homology and sodA and recN phylogenies. Vet. Microbiol. 162(2-4), 842-849 (2013).

12 Horby $\mathrm{P}$, Wertheim $\mathrm{H}, \mathrm{Ha} \mathrm{NH}$ et al. Stimulating the development of national Streptococcus suis guidelines in Vietnam through a strategic research partnership. Bull. World Health Organ. 88(6), 458-461 (2010).

13 Nghia HD, Tu Le TP, Wolbers M et al. Risk factors of Streptococcus suis infection in Vietnam. A case-control study. PLoS ONE 6(3), e17604 (2011).

- Identification of most important risk factors associated with $S$. suis bacterial meningitis in parts of Asia.

14 Takeuchi D, Kerdsin A, Pienpringam A et al. Population-based study of Streptococcus suis infection in humans in Phayao Province in northern Thailand. PLoS ONE 7(2), e31265 (2012).

15 Kerdsin A, Dejsirilert S, Puangpatra P et al. Genotypic profile of Streptococcus suis serotype 2 and clinical features of infection in humans, Thailand. Emerg. Infect. Dis. 17(5), 835-842 (2011).

- Identification of the most important S. suis sequence types in human infections in Thailand.

16 Schultsz C, Jansen E, Keijzers W et al. Differences in the population structure of invasive Streptococcus suis strains isolated from pigs and from humans in The Netherlands. PLoS ONE 7(5), e33854 (2012). 